DEMO-HEP-96/03

September 1996

\title{
Nullification in scalar theories with derivative couplings
}

\author{
Ernestos N. Argyres ${ }^{\dagger}$, and Costas G. Papadopoulos ${ }^{\ddagger}$

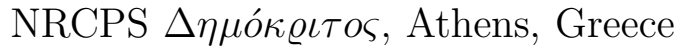 \\ Maarten Bruinsma* \\ NIKHEF, Amsterdam, the Netherlands \\ Ronald Kleiss ${ }^{\star \star}$ \\ University of Nijmegen, Nijmegen, the Netherlands
}

\begin{abstract}
We discuss the structure of scalar field theories having the property that all on-shell $S$-matrix elements vanish in tree approximation. It is shown that there exists a large class of such theories, with derivative couplings, which are all locally related to a free theory by a nonlinear transformation. It is also shown that a field-dependent wave-function renormalization provides all necessary counterterms so that all onshell $S$-matrix elements vanish also at the one-loop level.
\end{abstract}

E-mail: †argyres@cyclades.nrcps.ariadne-t.gr, `Costas.Papadopoulos@cern.ch, `t26@nikhefh.nikhef.nl,,^kleiss@sci.kun.nl. 


\section{Introduction}

\subsection{Threshold nullification in scalar theories}

The last few years, there has been considerable interest in the high-multiplicity predictions of scalar field theories [1]. Originally motivated by considerations on the possible enhancement of the rate of nonperturbative $B+L$-violating processes [2], the study of multiparticle production in perturbation theory has led to several new insights. In the first place, it has been proven that, at least in the tree approximation, the cross section will violate the bounds imposed by unitarity when the multiplicity and energy become sufficiently high [3, 4. Since the kinematics of many-particle final states can become extremely involved, most exact results have been derived for situations where all final-state particles are produced at rest. In addition, results have been derived for the case where one or two final-state particles are off-shell. These can be used in the evaluation of one- and two-loop corrections [5]. Another, and potentially much more interesting, phenomenon is that of threshold nullification [6, 7], which consists of the following. In some scalar theories, the threshold amplitudes $\mathcal{A}(2 \rightarrow n)$ for the $2 \rightarrow n$ process vanish identically for an infinite number of values of $n$. For instance, a pure $\phi^{3}$ theory leads to $\mathcal{A}(2 \rightarrow n)=0$ for all $n>3$; for a pure $\phi^{4}$ theory the same happens for $n>4$, and in a spontaneously broken $\phi^{4}$ theory even for $n>2$. Other, nonrenormalizable theories are known showing related behaviour. Apart from a relation between nullification and integrability of the classical action [8], no real physical reason for threshold nullification is evident. At any rate, above the kinematical threshold such theories do not show exact nullification [9], except for integrable theories in two dimensions, as for example in the sine-Gordon theory.

\subsection{The Ooguri-Vafa vertex}

Several years ago, Ooguri and Vafa discussed the geometry of $N=2$ strings, in which they discovered a similar nullification property also beyond the threshold [10]. They illustrated this string theory by a self-interacting scalar field theory defined on a space with a $(1,1,-1,-1)$ metric. Let each momentum $p$ be denoted by four real components, $\left(p^{1}, p^{2}, p^{3}, p^{4}\right)$, or, equivalently, by two complex ones, $\left(p^{1}+i p^{2}, p^{3}+i p^{4}\right)$. The massless scalar theory is then defined by the following three-point interaction vertex:

$$
\left(p_{1} \cdot \bar{p}_{2}\right)\left(p_{2} \cdot \bar{p}_{1}\right)-\left(p_{1} \cdot \bar{p}_{1}\right)\left(p_{2} \cdot \bar{p}_{2}\right)
$$

where $p_{1,2}$ are two of the momenta incoming into the vertex. It can be checked that, indeed, this theory has the property that all tree-level $S$-matrix elements with more than 3 external legs vanish identically [11]. In this $2+2$-dimensional spacetime, a massless particle can decay into two massless ones, so that the 3 -point $S$-matrix element is also physical. It is, however, not invariant under general boosts: the symmetry of the vertex is only $U(1,1)$ instead of $S O(2,2)$ [12, casting therefore some doubt on the usefulness of this effective theory as a physically meaningful one, 
even in $2+2$ dimensions. A systematic search through a large class of three-point vertices with four derivatives suggests that no Lorentz-invariant theory with 'total nullification' can be constructed from three-point interactions with four derivatives alone.

\section{Tree-order Amplitudes}

\subsection{The recursion relation}

Motivated by the previous analysis we are studying theories with two-derivative couplings. In these theories the vertex, with $k$ external scalar legs, is given by

$$
-i \lambda_{k}\left(p_{1}^{2}+p_{2}^{2}+\cdots+p_{k}^{2}\right)-i m^{2} \mu_{k}
$$

where $p_{1}, \ldots, p_{k}$ are the momenta in the legs, and $m$ is the mass of the scalar field. Notice that this is the most general Lorentz invariant and Bose symmetric vertex. The dimensionality of both $\lambda_{p}$ and $\mu_{p}$ is $2-p$. We shall allow for an arbitrary number of such couplings, and write

$$
\begin{aligned}
V(\phi) & =\sum_{p \geq 3} \frac{\lambda_{p}}{p !} \phi^{p}, \\
W(\phi) & =\sum_{p \geq 3} \frac{\mu_{p}}{p !} \phi^{p}, \\
\Omega(\phi) & =\frac{1}{2} m^{2} \phi^{2}+m^{2} W(\phi) ;
\end{aligned}
$$

The corresponding Lagrangian reads, of course,

$$
\mathcal{L}=\frac{1}{2}\left(\partial_{\mu} \phi\right)^{2}\left(1-2 V^{(2)}(\phi)\right)-\Omega(\phi)
$$

We shall compute the tree-level Green's functions with $(n+1)$ legs, $n$ of which have on-shell momenta $p_{1}, \ldots, p_{n}$, with $p_{1}^{2}=\cdots=p_{n}^{2}=m^{2}$, and the remaining one offshell momentum is $p_{0}$, with $p_{0}^{2}=s$. The on-shell legs are all truncated, but in the leg with momentum $p_{0}$ the full propagator is kept. We denote the one-leg-untrancated amplitude by $\mathcal{A}_{n}$. It is easy to see that $\mathcal{A}_{0}=0$, and $\mathcal{A}_{1}=1$. The recusrion relation for $n>1$ reads, Fig.1,

$$
\begin{aligned}
\mathcal{A}_{[n]}=\sum_{p \geq 2} \frac{1}{p !\left(s-m^{2}\right)} \sum_{\left[n_{1}\right], \ldots,\left[n_{p}\right]} \mathcal{A}_{\left[n_{1}\right]} \cdots \mathcal{A}_{\left[n_{p}\right]} \\
\left(\lambda_{p+1}\left[s+s_{\left[n_{1}\right]}+\cdots+s_{\left[n_{p}\right]}\right]+m^{2} \mu_{p+1}\right),
\end{aligned}
$$

where

$$
s_{[n]}=p_{[n]}^{2} \text { and } p_{[n]}^{\mu}=\sum_{i \in[n]} p_{i}^{\mu} .
$$

Here, we used the following notation. The set $\left[k_{i}\right]$ is a set of $k_{i}$ distinct labels taken from $[n]=\{1,2,3, \ldots, n\}$. In the recursion relation, the sets $\left[k_{1}\right],\left[k_{2}\right], \ldots,\left[k_{p}\right]$ do not intersect and form a complete partition of $[n]$. 


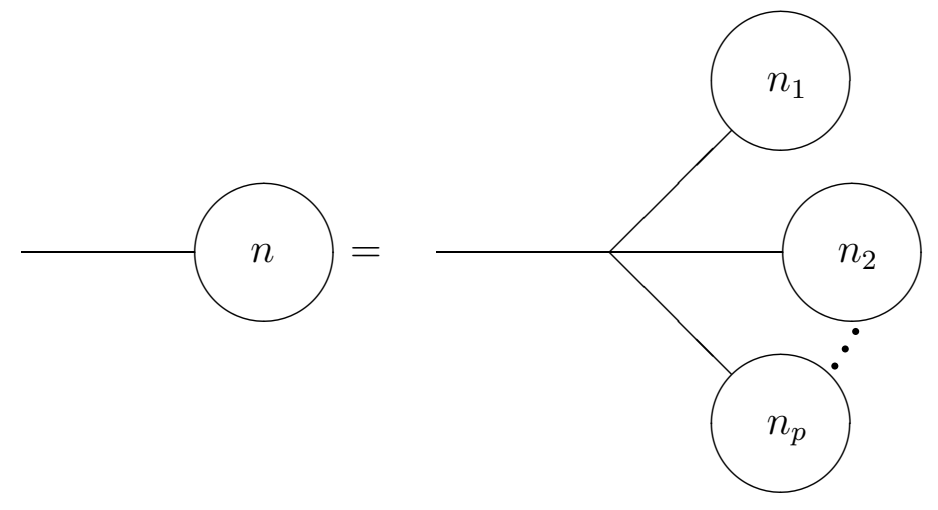

Figure 1: Diagrammatic representation of the recursion formula for the tree-order amplitudes.

\subsection{Conditions on the potentials}

It is easily seen that, if the $\mathcal{A}_{[k]}$ are pure numbers for $k<n$, then $\mathcal{A}_{[n]}$ will only depend on $s$ and $m^{2}$, and not on any other dot products, because of the symmetry of the sum over subsets:

$$
\begin{aligned}
& \sum^{\prime}\left(s+s_{\left[n_{1}\right]}+\cdots+s_{\left[n_{p}\right]}\right)= \\
& \left(s+n m^{2}\right) \frac{n !}{n_{1} ! \cdots n_{p} !}+\left(s-n m^{2}\right)\left\{\frac{(n-2) !}{\left(n_{1}-2\right) ! \cdots n_{p} !}+\ldots+\frac{(n-2) !}{n_{1} ! \cdots\left(n_{p}-2\right) !}\right\},
\end{aligned}
$$

where the sum is performed over all subsets $\left[n_{1}\right], \ldots,\left[n_{p}\right]$ with $n_{1}, \ldots, n_{p}$ fixed. If in addition we manage to make $\mathcal{A}_{n}$ also independent of $s$ and $m^{2}$, then the $S$-matrix elements will vanish upon truncation of the remaining leg, and we have what we call total nullification. We may therefore assume the $\mathcal{A}_{[n]}$ to be all independent of both $s$ and $m$, and examine to what requirements this leads on the relation among the potentials $V(\phi)$ and $W(\phi)$. Therefore, we put $\mathcal{A}_{[n]}=\mathcal{A}_{n}$, and find after some combinatorics

$$
\begin{aligned}
\left(s-m^{2}\right) \mathcal{A}_{n}= & \sum_{p \geq 3} \frac{1}{p !} \sum_{n_{1}, \ldots, n_{p}} \mathcal{A}_{n_{1}} \cdots \mathcal{A}_{n_{p}} \\
& {\left[\left(\lambda_{p+1}\left(s+n m^{2}\right)+m^{2} \mu_{p+1}\right) \frac{n !}{n_{1} ! n_{2} ! \cdots n_{p} !}\right.} \\
& \left.+\lambda_{p+1}\left(s-n m^{2}\right) \frac{p(n-2) !}{\left(n_{1}-2\right) ! n_{2} ! \cdots n_{p} !}\right] .
\end{aligned}
$$

As usual, we define a generating function as

$$
f(z)=\sum_{n>0} \mathcal{A}_{n} \frac{z^{n}}{n !},
$$


so that $f(0)=0$ and $f^{\prime}(0)=1$. Our recursion relation then becomes the following differential equation, with $\partial \equiv \partial / \partial z$ :

$$
\begin{aligned}
\left(s-m^{2}\right) f= & \left(s-m^{2}\right) z+\left(s+m^{2} z \partial\right) V^{(1)} \\
& +\left(s-m^{2} z \partial\right) \partial^{-2}\left(f^{\prime \prime} V^{(2)}\right)+m^{2} W^{(1)}
\end{aligned}
$$

with $\partial^{-2}$ defined by

$$
\partial^{-2} F=G \Leftrightarrow G=\partial^{2} F
$$

and

$$
V^{(n)}=\frac{\partial^{n} V}{\partial f^{n}}
$$

Now, since the $\mathcal{A}_{n}$ are by assumption independent of $s$ and $m^{2}$, we may take $m=0$ in the above equation and apply $\partial^{2}$, to end up with

$$
f^{\prime \prime}=2 f^{\prime \prime} V^{(2)}+\left(f^{\prime}\right)^{2} V^{(3)} .
$$

It is easily found that $f=f(z)$ and $z=\omega(f)$ must then be related by

$$
z=\omega(f)=\int_{0}^{f} d x \sqrt{1-2 V^{(2)}(x)} .
$$

Inserting this result in the $m^{2}$-dependent part of Eq.(8), we then find that, in order to arrive at total nullification, $W(f)$ must satisfy

$$
W(f)=-\frac{1}{2} f^{2}+\frac{1}{2} \omega(f)^{2} .
$$

Notice that in a massless theory total nullification exists for arbitrary 'potential' $V(\phi)$, whereas in the massive case the potential $W(\phi)$ is related to $V(\phi)$ through Eq.(11). Moreover, if we rewrite our Lagrangian in terms of the 'composite' field $\omega(\phi)$ instead of $\phi$, we obtain

$$
\mathcal{L}=\frac{1}{2}\left(\partial_{\mu} \omega\right)^{2}-\frac{1}{2} m^{2} \omega^{2}
$$

We see that, on the basis of this nonlinear transformation, all scalar theories with total nullification at tree order become locally equivalent to the free theory. It is also easy to verify that the generating function is a solution of the classical field equations [13.

\section{One-loop Amplitudes}

\subsection{The one-loop recursion relation}

Although the theory for the field $\phi(x)$ as defined by Eq.(3) seems to be related, at least locally, to a free field theory of the 'composite' field $\omega$, one might expect 


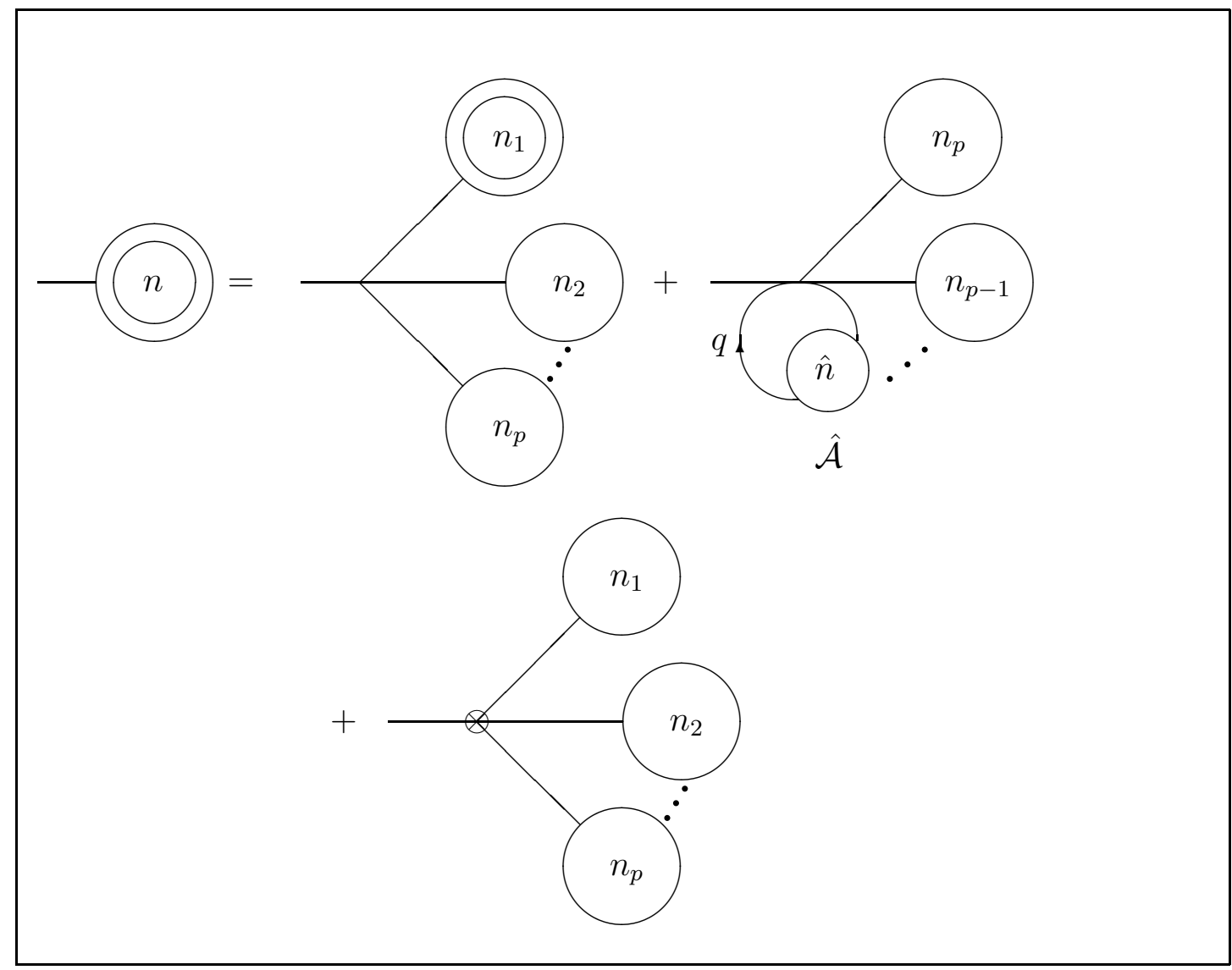

Figure 2: Diagrammatic representation of the recursion formula for the one-loop graphs (two circles). The single circles are the tree-order amplitude. The blob with two external legs corresponds to $\hat{\mathcal{A}}$, as explained in the text.

that this is a classical result and at the one-loop level the two theories will be very different. This reasoning is further supported by the fact that the theory of Eq.(3) is not renormalizable by the standard power-counting criterion.

Nevertheless, we have found that all one-loop divergencies of the one-leg-untruncated amplitudes $\mathcal{A}_{n}^{L}$, are indeed canceled by counterterms which can be cast into a form identical to the bare lagrangian Eq.(3), using a non-linear wave-function renormalization. Let us be more specific and write down the one-loop recursion relation corresponding to Fig.2:

$$
\left(s-m^{2}\right) \mathcal{A}_{[n]}^{L}=\mathcal{P}_{1}+\mathcal{P}_{2}+\mathcal{P}_{3}
$$

where $\mathcal{P}_{1}$ is the homogeneous term,

$$
\begin{aligned}
\mathcal{P}_{1}= & \sum \mathcal{A}_{\left[n_{1}\right]}^{L} \mathcal{A}_{n_{2}} \cdots \mathcal{A}_{n_{p}} \frac{1}{(p-1) !} \\
& {\left[\left(s+s_{\left[n_{1}\right]}+\ldots+s_{\left[n_{p}\right]}\right) \lambda_{p+1}+m^{2} \mu_{p+1}\right] }
\end{aligned}
$$


$\mathcal{P}_{2}$ is the inhomogeneous term,

$$
\begin{aligned}
\mathcal{P}_{2}= & \frac{1}{2} \sum \int \frac{d^{D} q}{(2 \pi)^{D}}(-i) \hat{\mathcal{A}}_{q[\hat{n}]} \mathcal{A}_{n_{3}} \ldots \mathcal{A}_{n_{p}} \frac{1}{(p-2) !} \\
& {\left[\left(s+\left(q+p_{[\hat{n}]}\right)^{2}+s_{\left[n_{3}\right]}+\ldots+s_{\left[n_{p}\right]}\right) \lambda_{p+1}+m^{2} \mu_{p+1}\right] }
\end{aligned}
$$

and $\mathcal{P}_{3}$ is the counterterm contribution, to be determined,

$$
\begin{aligned}
\mathcal{P}_{3}= & \sum \mathcal{A}_{n_{1}} \mathcal{A}_{n_{2}} \cdots \mathcal{A}_{n_{p}} \frac{1}{p !} \\
& {\left[\left(s+s_{\left[n_{1}\right]}+\ldots+s_{\left[n_{p}\right]}\right) \hat{\lambda}_{p+1}+m^{2} \hat{\mu}_{p+1}\right] } \\
+ & \text { tadpole contributions. }
\end{aligned}
$$

The one-loop counterterms can be written as

$$
\hat{V}(\phi)=\sum_{p \geq 3} \frac{\hat{\lambda}_{p}}{p !} \phi^{p} \text { and } \hat{W}(\phi)=\sum_{p \geq 3} \frac{\hat{\mu}_{p}}{p !} \phi^{p} .
$$

\subsection{Two-leg-untruncated amplitudes}

In order to proceed with Eq.(13) on has to calculate $\hat{\mathcal{A}}_{q[n]}$, which is the amplitude with two untruncated external legs at tree order. Since, by dimensional analysis, the dependence on the energy will be of the form $E^{-2}$, we make the ansatz that

$$
\hat{\mathcal{A}}_{q[n]}=\sum_{[k] \subset[n] \cup\{q\}} C_{[k]} \frac{i}{s_{[k]}-m^{2}} .
$$

The next step is to consider the limit $s_{[k]}-m^{2} \rightarrow 0$ and try to identify the residues of these simple poles. This can be done diagrammatically, by cutting the

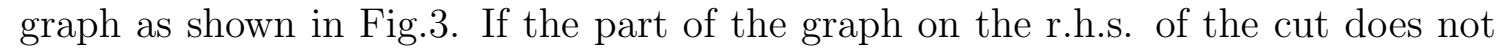
include the momentum $q$, all legs of this subgraph are on-shell, and according to our previous results its contribution vanishes. The only non-vanishing contribution comes, from the r.h.s. subgraphs which include the momentum $q$, and the result is simply given by:

$$
\hat{\mathcal{A}}_{q[n]}=\sum_{n_{1}+n_{2}=n} \mathcal{A}_{n_{1}+1} \mathcal{A}_{n_{2}+1} \sum_{\left[n_{1}\right]} \frac{i}{\left(q+p_{\left[n_{1}\right]}\right)^{2}-m^{2}} .
$$




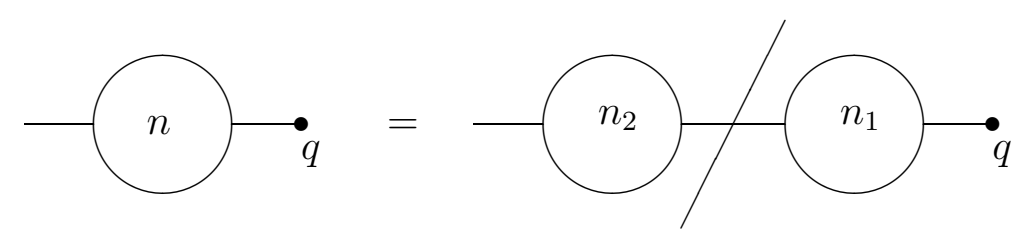

Figure 3: Diagrammatic representation of the recursion relation for the amplitude with two untruncated external legs.

\subsection{One-loop renormalization}

Using now Eq.(19) and performing the shift $q \rightarrow q-p_{\left[n_{1}\right]}$ the second term of the r.h.s of Eq.(13) is written as:

$$
\begin{aligned}
\mathcal{P}_{2}= & \frac{1}{2} \sum \int \frac{d^{D} q}{(2 \pi)^{D}} \frac{1}{q^{2}-m^{2}} \mathcal{A}_{n_{1}+1} \mathcal{A}_{n_{2}+1} \mathcal{A}_{n_{3}} \ldots \mathcal{A}_{n_{p}} \frac{1}{(p-2) !} \\
& {\left[\left(s+2 q^{2}+s_{\left[n_{1}\right]}+\ldots+s_{\left[n_{p}\right]}\right) \lambda_{p+1}+m^{2} \mu_{p+1}\right] . }
\end{aligned}
$$

It is now easy to see that in dimensional regularization only one form of divergence $^{1}$ appears, namely

$$
\mathcal{I}=\int \frac{d^{D} q}{(2 \pi)^{D}} \frac{1}{q^{2}-m^{2}}
$$

so the previous equation can be cast into the form:

$$
\begin{aligned}
\mathcal{P}_{2}= & \frac{1}{2} \mathcal{I} \sum \mathcal{A}_{n_{1}+1} \mathcal{A}_{n_{2}+1} \mathcal{A}_{n_{3}} \ldots \mathcal{A}_{n_{p}} \frac{1}{(p-2) !} \\
& {\left[\left(s+2 m^{2}+s_{\left[n_{1}\right]}+\ldots+s_{\left[n_{p}\right]}\right) \lambda_{p+1}+m^{2} \mu_{p+1}\right] . }
\end{aligned}
$$

In order to calculate the counterterms needed to cancel the divergencies of the one-loop amplitudes, we use the generating function technique by summing the inhomogeneous part, $\mathcal{P}_{2}+\mathcal{P}_{3}$ of Eq.(13) after multiplying by $z^{n} / n$ !. This can be done because all amplitudes $\mathcal{A}$ appearing in the inhomogeneous part are pure numbers, i.e. they do not depend on any specific product $p_{i} \cdot p_{j}$. The result is

$$
\sum\left(\mathcal{P}_{2}+\mathcal{P}_{3}\right) \frac{z^{n}}{n !}=\frac{1}{2} \mathcal{I}\left\{\left(s+2 m^{2}+m^{2} z \partial\right) f^{\prime 2} V^{(3)}\right.
$$

\footnotetext{
${ }^{1}$ This is also true in the Pauli-Villars regularization method.
} 


$$
\begin{aligned}
& \left.+\left(s-m^{2} z \partial\right) \partial^{-2}\left[2 f^{\prime \prime \prime} f^{\prime} V^{(3)}+f^{\prime 2} f^{\prime \prime} V^{(4)}\right]+m^{2} f^{\prime 2} W^{(3)}\right\} \\
& +\left(s+m^{2} z \partial\right) \hat{V}^{(1)}+\left(s-m^{2} z \partial\right) \partial^{-2}\left[f^{\prime \prime} \hat{V}^{(2)}\right]+m^{2} \hat{W}^{(1)} \\
& + \text { tadpole contributions }
\end{aligned}
$$

The 'renormalization' is now performed in the following sense: starting with the bare lagrangian

$$
\mathcal{L}=\frac{1}{2}\left(\partial_{\mu} \phi_{0}\right)^{2}\left(1-2 V^{(2)}\left(\phi_{0}\right)\right)-\frac{1}{2} m^{2} \phi_{0}^{2}-m^{2} W\left(\phi_{0}\right)
$$

we define $\phi_{0}=Z^{1 / 2} \phi$, with $Z=1+\mathcal{I}^{\prime} h(\phi)$ where

$$
h(\phi)=\frac{f^{\prime \prime}}{f}+\frac{f^{\prime}}{f}(b-2 c z) .
$$

Notice that the r.h.s. of Eq.(24) is expressed as a function of $z$, but $z$ should be understood as a function of $\phi$, given by $z=\omega[\phi]$ (see Eq.(10)). Moreover the infinite subtraction constant $\mathcal{I}^{\prime}$ is such that $\mathcal{I}^{\prime}-\mathcal{I}=$ finite. This finite difference between $\mathcal{I}^{\prime}$ and $\mathcal{I}$ is of course attributed to the subtraction scheme. In order to avoid any unnecessary, for the moment, complication let us choose $\mathcal{I}^{\prime}=\mathcal{I}$, which corresponds to a total subtraction scheme: this is possible since only one form of divergence, proportional to $\mathcal{I}$, appears in the Eq.(22). Then, expanding Eq.(23) and keeping linear terms in $\mathcal{I}$, we obtain

$$
\mathcal{L}=\frac{1}{2}\left(\partial_{\mu} \phi\right)^{2}\left(1-2 V^{(2)}(\phi)-2 \hat{V}^{(2)}(\phi)\right)-\frac{1}{2} m^{2} \phi^{2}-m^{2} W(\phi)-m^{2} \hat{W}(\phi),
$$

where

$$
\hat{V}^{(2)}=\mathcal{I}\left(\frac{1}{2} \frac{f^{\prime \prime 2}}{f^{\prime 4}}-\frac{1}{2} \frac{f^{\prime \prime \prime}}{f^{\prime 3}}+c \frac{1}{f^{\prime 2}}\right)
$$

and

$$
\hat{W}=\mathcal{I}\left(\frac{b}{2} z-c z^{2}+\frac{z}{2} \frac{f^{\prime \prime}}{f^{\prime}}\right) .
$$

The dimensionful constants $b$ and $c$ are determined by the requirement that $\hat{V}$ and $\hat{W}$, start with $\phi^{3}$.

After some algebraic effort ${ }^{2}$ one can see that the expression Eq.(22) becomes free of divergencies, even more becomes identically null, if the 'tadpole' contributions are given by

$$
\frac{b}{2} m^{2} \mathcal{I} \frac{1}{f^{\prime}}
$$

which is nothing but the 'tadpole' counterterm of the free theory

$$
\delta \mathcal{L}=\frac{b}{2} m^{2} \mathcal{I} \omega .
$$

\footnotetext{
${ }^{2}$ We have verified the results using Mathematica
} 
Having succeeded in canceling the divergencies, one can easily see from Eq. (13) that $\mathcal{A}_{n}^{L}=0$ : this is because the recursion relation is linear with respect to $\mathcal{A}_{n}^{L}$ and

moreover $\mathcal{A}_{0}^{L}=\mathcal{A}_{1}^{L}=0$. Notice that, in the total subtraction scheme, there is no mass renormalization at the one-loop level.

Of course in general the renormalization of this theory, even at the one-loop approximation, is actually much more involved and we are not going to further continue the discussion in this paper. For instance one might consider renormalization of some coupling constants introduced by the potentials $V(\phi)$ and $W(\phi)$, so that knowledge of their specific form is necessary. Nevertheless our results suggest that, although the theory defined by Eq.(3) is not power-counting renormalizable, divergencies of the one-leg-untruncated amplitudes can be canceled by appropriate counterterms, as we have explicitly proven at the one-loop level. Moreover these counterterms can be cast into a form that is identical to the bare lagrangian, and hence equivalent to the free $\omega$-field theory, by using a field-dependent wave-function renormalization as well as the 'tadpole' counterterm of the free theory. What actually happens is the following: since the theory is not power-counting renormalizable divergencies of a generic Green's function are not canceled by the counterterms given in Eq.(26,27). Nevertheless their combination corresponding to the one-leg-untruncated amplitude are free of divergencies. In that sense, one-leg-untruncated amplitudes as well as the on-shell $S$-matrix elements are ultraviolet finite quantities. Moreover, in a total subtraction scheme, they are all vanishing! This total nullification seems to be related to the existence of the nonlinear transformation $\phi_{0} \rightarrow \omega\left[\phi_{0}\right]$, which transforms the bare interacting lagrangian into the free one. It is not inconceivable that the equivalence between the two formulations, i.e. in $\phi$ and $\omega$, as far as the $S$-matrix elements are concerned, may be extended to all orders in perturbation theory.

\section{Discussion}

In order to get some more insight on the physics issues, let us recall the example of a $\Phi^{4}$ theory with spontaneously-broken reflection symmetry, which after the appropriate shift, $\Phi=\phi+v$, with $v=\langle\Phi\rangle$, takes the form:

$$
\mathcal{L}=\frac{1}{2}\left(\partial_{\mu} \phi\right)^{2}-\frac{1}{2} m^{2} \phi^{2}\left(1+\frac{\phi}{2 v}\right)^{2} .
$$

The learned reader will recall that all $\mathcal{A}(2 \rightarrow n)$ threshold amplitudes vanish for $n \geq 3$. It is indeed surprising that the new theory resulting by simply adding a three- and four-point derivative couplings, namely

$$
\mathcal{L}=\frac{1}{2}\left(\partial_{\mu} \phi\right)^{2}\left(1+\frac{\phi}{v}\right)^{2}-\frac{1}{2} m^{2} \phi^{2}\left(1+\frac{\phi}{2 v}\right)^{2}
$$


exhibits the nullification property for all on-shell $]^{5} S$-matrix elements ! The one-leguntruncated amplitudes $\mathcal{A}_{n}$ are given by $(n \geq 2)$

$$
\mathcal{A}_{n}=(-v)^{1-n}(2 n-3) ! ! ~ .
$$

In fact the 'composite' field $\omega$ is given in this case by a simple polynomial relation:

$$
\omega=\phi\left(1+\frac{\phi}{2 v}\right)
$$

and has the same physical mass, $m$, as the elementary field. Quantum corrections make the above relation look more complicated, but still the theory possesses the total-nullification property.

Another interesting example is that of the sine-Gordon theory. The original lagrangian is given by

$$
\mathcal{L}=\frac{1}{2}\left(\partial_{\mu} \phi\right)^{2}-\frac{m^{2}}{\lambda^{2}}(1-\cos (\lambda \phi))
$$

The $\omega$-field is now defined by

$$
\omega[\phi]=\frac{2}{\lambda} \sin \frac{\lambda \phi}{2}
$$

and the new lagrangian is written as

$$
\mathcal{L}=\frac{1}{2}\left(\partial_{\mu} \phi\right)^{2} \cos ^{2}\left(\frac{\lambda \phi}{2}\right)-\frac{m^{2}}{\lambda^{2}}(1-\cos (\lambda \phi)) .
$$

Looking for finite energy stationary-solutions in $1+1$ dimensions we find that

$$
\phi(x)=\frac{1}{\lambda} \begin{cases}-2 \sin ^{-1}\left(e^{-m\left(x-x_{0}\right)}\right)+2 \pi & x \geq x_{0} \\ 2 \sin ^{-1}\left(e^{m\left(x-x_{0}\right)}\right) & x \leq x_{0} .\end{cases}
$$

The energy or the 'mass' of this solution is given by

$$
M_{\phi}=\frac{4 m}{\lambda^{2}}
$$

Moreover one can easily check that the virial theorem is indeed satisfied. Notice that the sine-Gordon soliton is given by

$$
\phi(x)=\frac{4}{\lambda} \tan ^{-1}\left(e^{m\left(x-x_{0}\right)}\right)
$$

and that the corresponding 'mass' is

$$
M_{s G}=\frac{8 m}{\lambda^{2}} .
$$


It is easy to see that both solutions, satisfy $\phi(-\infty)=0$ and $\phi(\infty)=2 \pi / \lambda$. In Fig. we show the energy-density profile of our solution, as well as that of the sine-Gordon soliton, for $m=1, \lambda=1$ and $x_{0}=0$.

What is the moral of this sine-Gordon-like example? The answer is more or less obvious: the new theory defined by Eq.(35), although it is locally equivalent to a free theory, by means of the transformation Eq.(34), it possesses new solutions, which do not exist in the free theory: it is well known that the only finite-energy solution of the free-theory is $\omega(x)=0$. This of course might have been suspected by the simple remark that the transformation Eq.(34) implies some kind of a global constraint on the field-magnitude; for instance if we restrict ourselves to real solutions then $|\omega(x)| \leq 2 / \lambda, \forall x$. Therefore theories with derivative couplings such as the ones studied in this paper are not trivially equivalent to the free theory.

Let us summarize our results by sketching the physical picture emerging from them. It is possible to consider scalar fields $\phi(x)$ with very complicated and highly non-trivial self-interactions described by Eq.(3). Nevertheless their apparent dynamics is such that it has no measurable effects: all $S$-matrix elements are vanishing (as we have explicitly proven up to the one-loop level). This total nullification is equivalent to the existence of a 'composite' field $\omega[\phi]$, whose dynamics, at least in

peturbation theory, is described by a locally free field theory: the $\omega$ field possesses no self-interactions.

\section{Acknowledgements}

C.G.P. would like to thank the Department of Physics of the Catholic University of Nijmegen, where part of this work was done, for its kind hospitality. This work was partially supported by the EU grant CHRX-CT93-0319.

\footnotetext{
${ }^{3}$ We would like to emphasize that nullification now extends over all physical phase-space.
} 


\section{References}

[1] M.B. Voloshin, 'Nonperturbative methods', talk given at 27th International Conference on High Energy Physics (ICHEP), Glasgow, Scotland, 20-27 Jul 1994, published in ICHEP 1994:121-134 (QCD161:H51:1994) and hep-ph/9409344, and references therein.

[2] A. Ringwald, Nucl.Phys. 330 (1990) 1.

[3] M. B. Voloshin, Nucl.Phys. B383 (1992) 233-248.

[4] E.N. Argyres, R. Kleiss and C.G. Papadopoulos, Nucl.Phys. B391(1993) 42-56 and Nucl.Phys. B391(1993) 57-68.

[5] E.N. Argyres, R. Kleiss and C.G. Papadopoulos, Phys.Lett. B308 (1993) 292296, ADDENDA-ibid. B319 (1993) 544;

M. B. Voloshin, Phys.Rev. D47 (1993) 357-361;

B. H. Smith, Phys.Rev. D47 (1993) 3518-3520.

[6] E.N. Argyres, R. Kleiss and C.G. Papadopoulos, Nucl.Phys. B395(1993) 3-16.

[7] M. B. Voloshin, Phys.Rev. D47 (1993) 2573-2577;

E.N. Argyres, R. Kleiss and C. Papadopoulos, Phys.Lett. B308 (1993) 315-321, ADDENDA-ibid. B319 (1993) 544;

B. H. Smith, Phys.Rev. D49 (1994) 1081-1085.

[8] E.N. Argyres, R. Kleiss and C.G. Papadopoulos, Phys.Lett. B302 (1993) 70-73, ADDENDA-ibid. B319 (1993) 544.

[9] C. G. Papadopoulos Phys.Lett. B324 (1994) 66-71.

[10] H. Ooguri and C. Vafa, Nucl.Phys.B361 (1991) 469.

[11] M. Bruinsma, doctorandus-thesis.

[12] N. Berkovits and C.Vafa, Nucl. Phys. B433 (1995) 123-180.

[13] L.S.Brown, Phys. Rev. D46 (1992) 4125-4127 


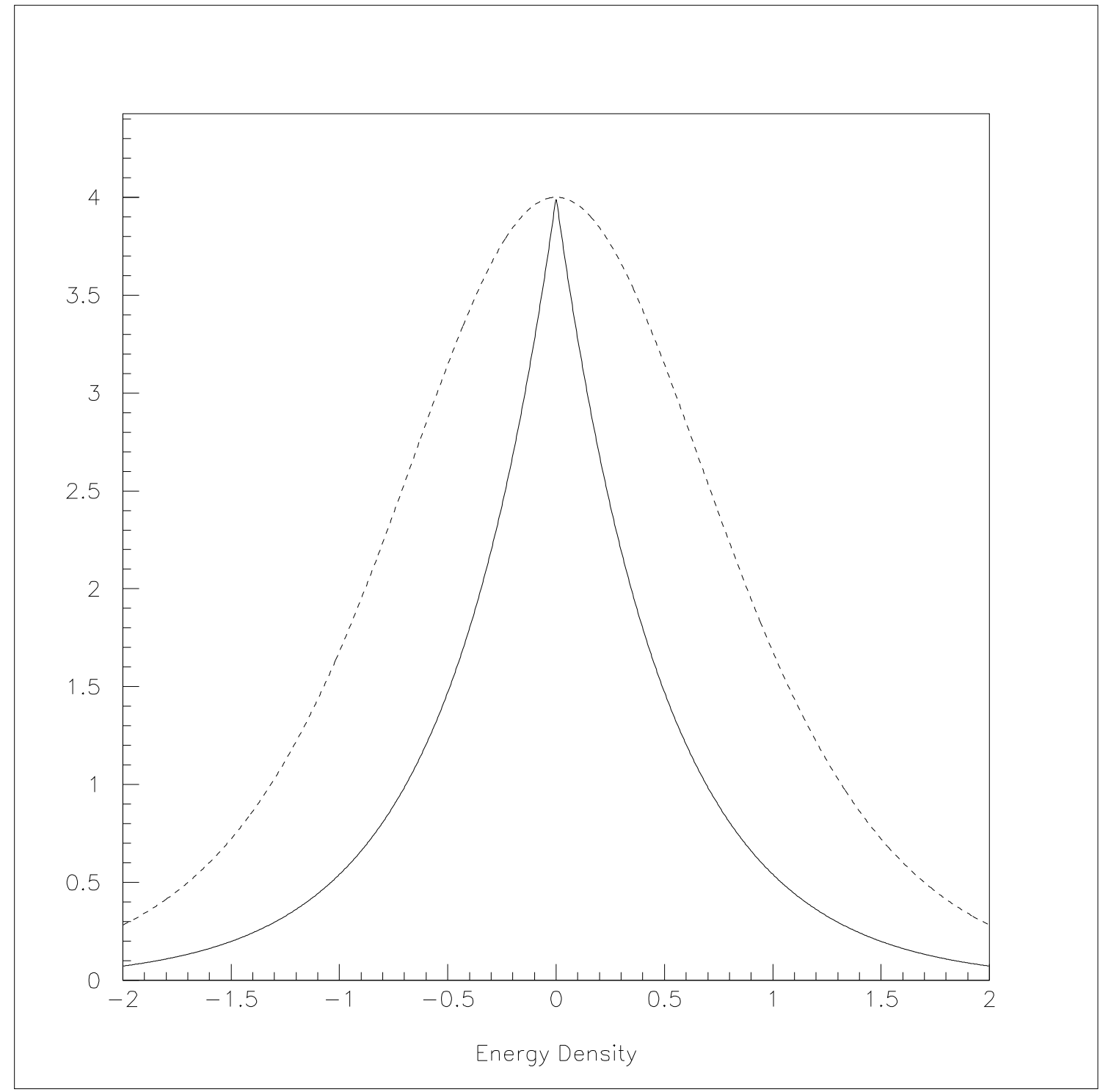

Figure 4: The energy density of the solution $\phi(x)$ given in Eq.(36). The dotted line represents the energy density of the sine-Gordon soliton-solution. 